Meta

Journal des traducteurs

Translators' Journal

\title{
Expériences et théorisation de la traduction littéraire en Chine
}

\section{$\mathrm{Xu}$ Jun et Liu Heping}

Volume 49, numéro 4, décembre 2004

URI : https://id.erudit.org/iderudit/009782ar

DOI : https://doi.org/10.7202/009782ar

Aller au sommaire du numéro

Éditeur(s)

Les Presses de l'Université de Montréal

ISSN

0026-0452 (imprimé)

1492-1421 (numérique)

Découvrir la revue

Citer cet article

Jun, X. \& Heping, L. (2004). Expériences et théorisation de la traduction littéraire en Chine. Meta, 49(4), 786-804. https://doi.org/10.7202/009782ar

\section{Résumé de l'article}

La traduction de la littérature étrangère a exercé une influence active et positive sur la littérature et la culture chinoises. Au cours du siècle passé, les traducteurs chinois ont accumulé de riches expériences et leurs réflexions sur leur activité ont ouvert une voie précieuse aux études théoriques de la traduction littéraire. Faisant le bilan de leurs expériences et réflexions sur la traduction littéraire, le présent article essaye de présenter brièvement des propos recueillis au cours de 18 entretiens avec 20 grands traducteurs, de dégager 10 problèmes fondamentaux relatifs à la traduction littéraire, d'en exploiter les valeurs théoriques et de fournir une possibilité de théorisation de la traduction littéraire. 


\title{
Expériences et théorisation de la traduction littéraire en Chine
}

\author{
XU JUN \\ Université de Nanjing, Nanjing, Chine \\ xyduo@publicl.ptt.js.cn \\ LIU HEPING \\ Université des langues et cultures de Beijing, Beijing, Chine \\ hepingliu@hotmail.com
}

\begin{abstract}
RÉSUMÉ
La traduction de la littérature étrangère a exercé une influence active et positive sur la littérature et la culture chinoises. Au cours du siècle passé, les traducteurs chinois ont accumulé de riches expériences et leurs réflexions sur leur activité ont ouvert une voie précieuse aux études théoriques de la traduction littéraire. Faisant le bilan de leurs expériences et réflexions sur la traduction littéraire, le présent article essaye de présenter brièvement des propos recueillis au cours de 18 entretiens avec 20 grands traducteurs, de dégager 10 problèmes fondamentaux relatifs à la traduction littéraire, d'en exploiter les valeurs théoriques et de fournir une possibilité de théorisation de la traduction littéraire.
\end{abstract}

\begin{abstract}
The translation of foreign literature has exerted an active and positive influence on Chinese literature and culture. In the past century, Chinese translators have accumulated rich experience, and their reflections on their practice constitute a precious perspective on the theoretical study of literary translation. Summarizing their experience and reflections on literary translation, the present essay tries to present the gist of 18 dialogues with 20 well-known Chinese translators on ten fundamental problems of literary translation. The paper aims at revealing the theoretical value of the dialogues and to offer a possibility of theorizing literary translation.
\end{abstract}

\section{MOTS-CLÉS/KEYWORDS}

expérience, théorisation, problèmes fondamentaux, traduction littéraire, traducteurs chinois

\section{Introduction}

La traduction littéraire en Chine peut remonter à l'époque des Six Royaumes (420534), mais l'introduction systématique de la littérature étrangère ne date que d'un siècle. Au cours du siècle qui vient de se terminer, la Chine a connu deux époques où la traduction littéraire était particulièrement florissante. La première se situait dans les années 1910 et 1920, et la deuxième dans les années 1980 et 1990. Plus de 8000 œuvres représentatives de presque 80 pays ont pu entrer en Chine, exerçant une influence active et positive sur la littérature et la culture chinoise. «La vitalité de la civilisation chinoise est due dans une large mesure à la traduction», a ainsi affirmé M. Ji Xianlin, traducteur renommé et professeur de l'Université de Pékin, en insistant sur le grand rôle joué par la traduction dans l'évolution de la société chinoise. Au 
cours des deux grandes périodes de traduction ci-dessus mentionnées, les traducteurs ont accumulé de riches expériences et leurs réflexions sur leur activité ont ouvert une voie précieuse aux études théoriques de la traduction littéraire.

Depuis ces dernières années, les chercheurs prêtent une attention particulière aux expériences et réflexions des grands traducteurs chinois. La classification, l'analyse, la synthèse de leurs expériences et réflexions ont fourni une possibilité de théorisation. C'est justement dans ces perspectives que s'inscrivent nos études sur la traduction littéraire à partir d'une série d'entretiens avec 20 traducteurs bien connus en Chine. Nous nous sommes fixé un but bien précis: à travers les entretiens avec les traducteurs, nous essayerons de répondre à deux questions qui nous paraissent essentielles. La première est de savoir si la traduction littéraire, activité essentiellement individuelle et créative, présente des traits communs à partir desquels on pourrait élaborer une théorie ou des théories. La deuxième question se pose de façon précise: quels sont les problèmes fondamentaux relatifs à la traduction littéraire? En répondant à ces deux questions, nous cherchons à faire le bilan des expériences de traducteurs et à les valoriser par voie de théorisation.

\section{Quelques mots sur le projet d'études}

Le projet d'études que nous avons élaboré a été proposé et approuvé par le ministère de l'Éducation supérieure de Chine, ce qui montre l'intérêt que portaient les membres du Conseil des sciences humaines chargés de l'évaluation du projet. Nos études se divisent en trois étapes.

Dans la première étape, le travail principal est de classer les réflexions des traducteurs chinois sur la traduction littéraire depuis le début $\mathrm{du} \mathrm{xx}^{\mathrm{e}}$ siècle. Nous avons été frappés à la fois par la richesse et la pauvreté de leurs réflexions: riches sont celles portées sur la technique et la manière de traduire, pauvres sont celles d'ordre théorique. Ainsi, les traducteurs parlaient-ils principalement du produit fini de leur activité en négligeant le processus et les facteurs influents de la traduction. La vision bornée au texte seul les empêchait très souvent de prendre conscience des facteurs extérieurs qui influaient plus ou moins sur leur acte de traduire. Partant chacun de leurs propres expériences, les traducteurs ont pourtant partagé dans beaucoup de cas les mêmes points de vue, notamment sur l'importance de la reproduction du style, la nécessité de la fidélité au texte d'origine, etc.

Le travail de classification nous a permis de nous rendre compte des traits particuliers et communs de leurs réflexions et de constater qu'une théorisation de la traduction littéraire ne peut se passer de précieuses valeurs que révèlent les expériences des traducteurs. Cette constatation nous amène à la deuxième étape de notre travail qui consiste à établir un questionnaire à partir de la base des écrits des traducteurs sur leur pratique.

L'établissement du questionnaire a pour but d'élargir l'horizon des traducteurs, d'approfondir leurs réflexions, d'en ressortir les points d'intérêt communs et d'en tirer des valeurs théoriques. Pour atteindre ce but, nous avons consacré beaucoup de temps pour classer, trier et analyser les écrits qui nous intéressaient sur le plan théorique. Et en lisant de nombreux écrits, nous nous sommes posé des questions telles que: Pourquoi les traducteurs, dans les années 1920 préféraient-ils les œuvres littéraires des pays sous-développés aux œuvres classiques universellement reconnues? 
Pourquoi beaucoup de traducteurs observaient-ils une attitude double à l'égard des œuvres de genres différents: fidélité pour les romans et liberté pour la poésie? Pourquoi certains traducteurs préféraient-ils le vrai au beau, et d'autres le beau au vrai? Quelle est la cause de cette contradiction? Pourquoi la fidélité au texte d'origine a-t-elle été considérée par beaucoup de gens comme une condition préalable pour assurer la bonne qualité de la traduction, tandis que le principe de l'élégance a provoqué une vive controverse parmi les traducteurs tout au long du siècle? Quels sont les obstacles pour reproduire le style de l'œuvre d'origine? La traduction littéraire a-t-elle contribué à l'enrichissement de la langue chinoise? Le traducteur est-il libre de choisir les œuvres à traduire? Quel est le souci principal du traducteur quand il se trouve embarrassé pour satisfaire à la fois l'auteur et le lecteur? Quelle est la relation entre la traduction et la création? Pourquoi en fonction des époques les traducteurs adoptentils des manières différentes de traduire? La liste de questions que nous avons établie est loin d'être close. L'idée nous est alors venue de réfléchir sur ces questions mêmes et d'essayer de les classer en types différents: le motif, le choix, le processus, les critères, les manières, les facteurs et le rôle de la traduction.

À partir de cette classification, nous sommes arrivés à la troisième étape : choisir les traducteurs représentatifs pour répondre aux questions en partant de leurs propres expériences et dégager de leurs réponses les problèmes fondamentaux relatifs à la traduction littéraire.

Nous avons d'abord fixé cinq critères de choix des traducteurs: premièrement, ils devaient avoir une longue expérience; deuxièmement, ils devaient jouir d'un renom national, même international; troisièmement, ils devaient représenter la traduction d'œuvres littéraires diverses écrites en langues de grande diffusion, représentatives par exemple de la littérature anglaise, française, espagnole, italienne, russe, japonaise, indienne, etc. ; quatrièmement, les traducteurs devaient avoir traduit de grands auteurs. Enfin, nous avons jugé préférable que les traducteurs choisis aient réfléchi sur la traduction et publié des articles à ce sujet. Selon ces critères, nous avons dressé la liste d'une trentaine de traducteurs, dont M. Fang Ping, traducteur de William Shakespeare; M. Jiang Feng, traducteur de Percy Bysshe Shelley; M.Yang Wuneng, traducteur de Johanne Wolfgang von Geothe; M. Li Wenjun, traducteur de Faulkner; M. Lu Tongliu, traducteur d'une trentaine d'œuvres italiennes aussi bien classiques que contemporaines; M. Lin Yi An, traducteur de la littérature espagnole et directeur de la traduction des Euvres complètes de Borges; M. Guo Hong An, traducteur de Baudelaire; M. Ji Xian Lin, traducteur de Valmiki et de Kalidasa de l'Inde; M. Cao Ying, traducteur d'une quarantaine d'œuvres classiques russes, en particulier celles de Léon Tolstoï... et deux couples de traducteurs reconnus en Chine, Xiao Qian et Wen Jie Ruo, cotraducteurs d'Ulysse; Zhao Ruihong et Yang Yi, traducteur de Stendhal, traductrice d'Emily Brontë. Les traducteurs choisis ont tous une expérience de plus d'un demi-siècle.

Hélas, avant même d'engager le dialogue avec les traducteurs classés dans notre liste, quatre d'entre eux nous ont malheureusement quittés pour toujours, laissant une lacune impossible à combler. Cinq sont décédés un an après les entretiens, dont Ye Junjian, traducteur des œuvres complètes de Hans Christian Andersen, et Li Mang, traducteur de la littérature japonaise. Les traducteurs cités ci-dessus, tous très âgés et riches en expériences, sont les plus respectés en Chine dans le milieu de la traduction. Les entretiens et les dialogues que nous avons réalisés avec eux ont été 
considérés par beaucoup comme «des travaux de sauvetage » consistant à préserver de précieuses réflexions de l'élite des traducteurs en Chine.

La liste une fois définie, nous nous sommes permis de nous adresser aux traducteurs en leur expliquant l'objectif de notre travail et en leur soumettant un questionnaire que nous avions établi sur la base d'une lecture attentive et d'une analyse approfondie de leurs écrits (y compris préface, postface, mots de traducteurs, etc.) sur la traduction. Notre travail a bénéficié de leur part d'un large soutien. Grâce à une participation active de ces traducteurs, et ce, malgré leur âge, nous avons pu élaborer durant trois ans, 18 dialogues qui nous ont permis d'engager des discussions approfondies sur la traduction et les problèmes relatifs à la traduction.

\section{Dialogues et problèmes théoriques dégagés}

Le projet d'études que nous avons ultérieurement décrit de façon sommaire a aussi bénéficié de l'aide de La forêt de traduction, revue bimensuelle concernant la traduction (avec un tirage de plus de 100000 exemplaires par numéro). Les propos recueillis au cours des dialogues ont été publiés par cette revue dans 18 numéros consécutifs (de 1998 à 2000). Pour nous, chaque dialogue engagé avec le traducteur était un processus de compréhension, d'études et de recherches. Tout au long de ce processus, nous approfondissions peu à peu nos études à partir du dialogue en face-à-face, par correspondance ou par téléphone. Nous n'oublierons jamais la voix faible mais passionnante de Ye Junjian, atteint d'un cancer, qui nous parlait au téléphone au dernier moment de sa vie. Les trois longues lettres que Xiao Qian, traducteur d'Ulysse, nous a fait parvenir de l'hôpital sont extrêmement précieuses. Dans ces lettres, Xiao Qian, gravement malade, nous a tracé la voie de traduction qu'il a parcourue durant plus de 60 ans et expliqué ce qu'il avait cherché à réaliser par la traduction, les principes auxquels il se tenait et le rôle que la traduction devait jouer dans les échanges culturels.

Le dialogue devenait très souvent un échange d'idées, et au fur et à mesure que le dialogue s'approfondissait, des points de vue différents s'affrontaient et suscitaient quelquefois un débat un peu houleux sur l'objectif et les manières de la traduction. Ainsi, M. Xu Yuanchong, très célèbre en Chine, traducteur en anglais et en français de la poésie classique chinoise et en chinois d'une vingtaine d'œuvres françaises, a-t-il insisté sur l'importance du beau dans la traduction littéraire, tandis que son interlocuteur prétendait que le vrai primait sur le beau. La confrontation d'idées et de points de vue a permis d'enrichir la discussion et de dégager des problèmes fondamentaux relatifs à la traduction littéraire.

Tout au long de nos études, nous avons constaté un paradoxe: la plupart des traducteurs affirmaient qu'il n'y avait pas de théories de la traduction et consentaient seulement à relater leurs expériences personnelles quand nous leur demandions de nous donner l'occasion d'explorer avec eux les problèmes théoriques de la traduction. Mais aux questions posées, ils ont tous exprimé leurs points de vue en les étayant d'exemples concrets et convaincants. Finalement, en relisant les propos recueillis, ils ont découvert qu'il y a non seulement une théorie, mais des théories, et qu'à la lumière de théories différentes, les manières de traduire s'avéraient différentes. "Le dialogue m'a permis de jeter un regard rétrospectif sur la voie que j'avais parcourue depuis plus d'un demi-siècle et de réfléchir sur les problèmes que j'avais rencontrés dans la pratique. Il est fort utile pour moi de faire le bilan de mes expériences et de voir par 
analyse ce qui est rationnel», a écrit Li Meng dans un article peu après la publication des propos recueillis dans La forêt de traduction.

En effet, grâce aux dialogues engagés nous avons eu l'occasion précieuse de connaître le grand esprit dont faisaient preuve les traducteurs qui nourrissaient tous un désir ardent de communiquer, de recréer, et d'apporter quelque chose d'utile à la culture de la langue d'arrivée par la traduction. Les riches expériences de ces traducteurs ont ouvert le champ d'horizon de nos études et nous ont fourni de précieux matériaux destinés à l'analyse par laquelle nous avons peu à peu pu voir se dessiner les grandes lignes de leurs idées et dégager les valeurs théoriques que recelaient leurs réflexions.

$\mathrm{Au}$ bout de trois ans de travail a pu voir le jour un livre intitulé: Théories et pratique de la traduction littéraire: dialogues et réflexions (voir Xu Jun 2001). En regroupant les 18 dialogues publiés dans La forêt de traduction, l'ouvrage a été consacré à la description de leurs expériences, à la classification de leurs idées, à la confrontation de leurs points de vue, à l'analyse des problèmes principaux de la traduction. Ainsi ont été dégagés 10 problèmes qui ont tous, selon nous, une valeur théorique. Qu'il nous soit permis de présenter en grandes lignes ces problèmes qui nous paraissent fondamentaux.

\subsection{La nature de la traduction}

Qu'est-ce que la traduction? Voilà une question à laquelle tout traductologue doit répondre de prime abord, puisque ce « qu'est-ce que» constitue une question de base pour tous les chercheurs de disciplines différentes. Cette question sur la nature de la traduction nous amènera à réfléchir sur sa définition, sur les problèmes qu'elle soulève avant de les théoriser.

La nature de la traduction peut se définir sous des angles divers: sous l'angle philosophique ou encore par son but, son objectif ou ses formes. À travers les réponses des vétérans chinois de la traduction lors de nos dialogues, nous pouvions constater que la plupart d'entre eux insistaient sur le but et le rôle qu'elle joue dans la communication lorsque nous demandions leurs réflexions sur la nature de la traduction.

Ji Xianlin indique: «La traduction est indispensable tant qu'il existe des échanges et une communication entre pays et nations différents. Ces échanges d'idées et de cultures seraient impossibles sans passer par la traduction, de même que le progrès du monde humanitaire risquerait d'être bloqué» (Ji Xianlin et Xu Jun 1998: 210). Cela revient à affirmer que la motivation de communication et d'échanges est l'origine de la traduction, en d'autres termes, elle doit sa naissance aux besoins des interlocuteurs de communautés linguistiques différentes.

Zhao Ruihong souligne lui aussi que la traduction est une communication intercommunautaire. Tant qu'il y aura des communautés de cultures différentes sur notre planète, les échanges culturels s'avèreront toujours nécessaires, d'où la nécessité de la traduction.

Aux yeux de Lu Xun, le plus grand écrivain chinois du $\mathrm{xx}^{\mathrm{e}}$ siècle, l'être humain préfere la compréhension réciproque aux barrières. La traduction littéraire est alors un des ponts servant aux échanges culturels (Zhao Yuihonh, Yan Yi et Xu Jun 1999: 209).

La traduction étant au service de la communication multilinguistique, elle est, elle aussi, un acte de communication, ceci malgré le changement de formes linguis- 
tiques du texte. Elle ne retransmet en effet un sens qu'en une forme linguistique autre que l'original. Ji Xianlin complète ainsi son idée par une métaphore:

si nous considérons la culture comme une rivière, nous pouvons effectivement remarquer que la rivière chinoise n'a jamais vu son lit tarir même s'il y avait des moments où l'eau lui manquait, car de l'eau fraîche ne cessait d'y être injectée, dont les deux sources les plus remarquables et abondantes de l'histoire sont celles venues de l'Inde, et de l'Europe par le canal de la traduction. La culture chinoise doit, selon moi, sa continuité et sa longévité à la traduction (JI Xianlin et Xu Jun 1998: 210).

Celle-ci joue non seulement un rôle non négligeable dans la communication, mais également dans le développement des cultures propres à chaque nation.

Il en est de même pour Ba Jin, auteur de Famille, printemps et automne: la traduction est une arme qui lui a été offerte, lui permettant de traverser l'obscurité avant l'arrivée du jour (Ba Jin 1996).

Les affirmations de ces traducteurs chinois renommés nous poussent à tirer une première conclusion: les traducteurs essayent dans la plupart des cas de comprendre et de définir la nature de la traduction par le but de la traduction: dans les années de guerre, la traduction était plutôt pour eux un moyen d'éveiller la conscience du peuple, de faire connaître les résistances menées par les peuples et de renforcer la conviction de la victoire. Tu An en témoigne par son vécu: "C’était au départ un amour que j'éprouvais pour les poèmes de Walt Whitman, or une grande admiration sur sa description poétique de la guerre de Sécession m'a finalement envahi. La publication de ma traduction était par conséquent très symbolique, puisque c'était un soutien que je pouvais apporter aux forces révolutionnaires représentées par Yan An, base de la révolution populaire chinoise de l'époque.»

\subsection{Les facteurs déterminant le choix des auvres à traduire}

La motivation et le but pèsent beaucoup sur la sélection des auteurs à traduire, mais le choix des œuvres dépend de plusieurs facteurs. Dans les premières années qui ont suivi la libération de la Chine en 1949, «être au service de la révolution et de la création littéraire» est alors un principe fondamental. C'est la raison pour laquelle beaucoup d'écrivains russes et des pays socialistes ont été introduits en Chine et en raison de ce principe primordial, le critère artistique ou esthétique cède naturellement sa place aux facteurs politiques et idéologiques. Léon Tolstoï et les idées humanistes véhiculées dans ses œuvres sont rendues en chinois grâce à Cao Ying et à ses 20 ans de traduction; Hans Christian Andersen, quant à lui, doit sa réputation à Ye Junjian: la sollicitude qu'Andersen nourrit, l'attention qu'il accorde à la dignité de l'homme et son exaltation pour le progrès du monde sont gravées dans la mémoire du large public chinois. Durant cette période, les œuvres européennes étaient en effet refusées, voire interdites. Plus tard, vers les années 1960, sont sortis des «livres jaunes» (couverture faite de papier jaune sans images) et certaines œuvres littéraires européennes ont pu voir le jour en Chine, publiés par l'Édition de la littérature du peuple. Pendant la Révolution culturelle (1966-1976), toute publication des œuvres littéraires venant de l'étranger est interrompue. Ce n'est qu'après la $3^{\text {e }}$ session du $11^{\mathrm{e}}$ Congrès du PCC (en 1978) que le printemps de la littérature revient dans le pays. Écoutons Fang Ping à propos des influences politiques négatives sur la traduction: «Lors de la Révolution culturelle, Le rouge et le noir, Le Père Goriot ainsi que d'autres 
œuvres similaires étaient violemment attaquées, seul William Shakespeare, lu et heureusement apprécié par Marx et Engles a fait exception à la règle. Dans un tel contexte idéologique, pratiquer la traduction, c'était comme aller rencontrer en cachette un amant contre la volonté des parents durant les dynasties féodales. »

La réforme et l'ouverture de la Chine vers la fin des années 1970 offrent l'occasion aux traducteurs de sélectionner les auteurs par critères esthétiques, culturels et artistiques. "Je ne traduis que ce que j'aime», nous révèle Xiao Qian quand il parle du choix de la traduction. Il a une forte passion pour les œuvres pleines d'ironie et de sarcasmes, de Fielding à Leacock. Tu An se distingue des autres en définissant ses principes de choix: premièrement, les œuvres poétiques les plus belles et reconnues dans l'histoire littéraire; deuxièment les œuvres qu'il aime et qui le touchent au cœur. Pour lui, «En introduisant au lecteur chinois les meilleurs poèmes, nous lui présentons "Vérité, vertu et beauté" de l'étranger. Je ne parviendrais jamais à une belle traduction sans amour pour les œuvres choisies.» Étant éditeur et traducteur, Tu An insistait sur l'importance d'une vision large et globale dans le choix des œuvres. Dans les années de paix, la valeur esthétique et littéraire primait dans le choix de la Maison d'Édition de la littérature du peuple. Cela dit, des œuvres étrangères intéressantes dont la valeur littéraire n'était pas forcément remarquable pouvaient également être prises en considération afin de couvrir les goûts divers du lecteur chinois (Tu An et Xu Jun 1999: 208).

Il est à souligner qu'en matière de choix des œuvres littéraires à traduire, c'est l'éditeur qui détient en quelque sorte le pouvoir de décision. Son choix est, hélas, souvent influencé par un facteur financier, en d'autres termes par les profits qui finissent parfois par primer sur l'esthétique. Le choix personnel du traducteur selon ses principes ou son goût sont par conséquent remis en question. Malgré cette constatation douloureuse, les opinions exprimées par les traducteurs chinois nous fournissent de précieux supports de réflexion en la matière.

\subsection{Les critères et principes de la traduction}

Nombreux sont ceux qui prétendent, depuis longtemps et aujourd'hui encore, qu'il n'existe pas de théorie de la traduction. Il s'ensuit d'une part que, la traduction n'est, pour eux, qu'un art, dont le résultat dépend complètement de la qualité, de l'appréhension et des connaissances du traducteur, d'autre part, que la pratique de la traduction ne nécessite pas, en l'occurrence, des critères et principes, encore moins une (des) théorie(s).

Nous pensons depuis toujours que la traduction est à la fois un acte de communication, un art réclamant sa propre loi et ses critères. Comment les traducteurs traduisent-ils? Par quels moyens parviennent-ils à la fidélité, à l'esprit et au style de l'œuvre originale? Quelle est la clef de la réussite dans leur traduction? «Fidélité, clarté et élégance», trois principes formulés par Yan Fu en 1898 sont, pendant longtemps en Chine, la bible de la pratique de la traduction. Or, les explications et la compréhension de ces principes sont tellement diverses que de grands débats sont lancés vers la fin des années 1980 et le début des années 1990 du siècle dernier dans le milieu traductologique chinois. Certains croient que ces principes entravent non seulement des recherches traductologiques mais bornent parallèlement les réflexions des traducteurs. Huang Shi Yu ne cache pas sa réprobation et dit clairement que, bien 
qu'Yan Fu ait joué un rôle incontestable dans l'introduction des idées nouvelles de l'étranger à l'époque, sa théorie traductologique est tout à fait fausse (Shen Suru 1998: 107). Certains autres les prennent comme principes de la traduction. Comme nous le savons, Yan Fu, en traduisant Theory of Evolution, se sent dépourvu et s'exclame: «Qu'il est difficile de respecter "fidélité, clarté et élégance" en traduction!» Il est clair que cette exclamation doit s'expliquer par les difficultés de la traduction, mais absolument pas par l'intention d'établir une théorie. Ces termes deviennent trois principes directeurs de la traduction suite aux interprétations des traducteurs qui les associent à leur compréhension de la traduction. La valeur de la théorie réside, selon Luo Xinzhang, dans son rôle directeur pour la pratique. Ye Junjian partageant son opinion, considère que les principes de Yan Fu sont pratiques, scientifiques et faciles à maîtriser. Il prétend même qu'ils peuvent devenir une norme internationale, applicable à la traduction entre tous les couples de langues. Si l'on peut bien mesurer et appliquer ces trois principes dans la traduction, on pourra éviter toute erreur éventuelle (Ye Junjian et Xu Jun 1998: 202).

$\mathrm{Tu}$ An ne nous cache rien quand il dit:

J'ai foi en «fidélité, clarté et élégance» de Yan Fu. Parmi ces trois principes, je pense que la fidélité est primordiale et la clef de voûte du problème. Les trois principes de Yan Fu sont comparables aux trois principes de l'existence de l'homme: 'le vrai, le bon et le beau'. Le vrai est l'essentiel, le bon sa dénotation et le beau sa connotation. Sans le vrai, il n'est plus question du bon et ni du beau... Il en est de même pour les principes de Yan Fu: sans fidélidé, clarté et élégance n’ont plus de sens... (Tu An et Xu Jun 1999: 202).

La révélation des traducteurs nous fait découvrir quelque chose d'universel dans leur pratique. Consciemment ou inconsciemment, beaucoup de traducteurs riches en expériences créent leurs propres principes/critères de la traduction sur le chemin d'exercice de leur métier (Li Mang et Xu Jun 2000 : 204). Li Mang nous explique sa compréhension de «l'élégance»: la fidélité se traduit par le contenu et le style de la traduction; la clarté exige du traducteur littéraire une fluidité d'expression. Comme la clarté et l'élégance appartiennent au niveau stylistique du texte, l'écart devient grand et évident au sujet du style entre les traducteurs de niveaux différents ( $\mathrm{Li}$ Mang et Xu Jun 2000: 205).

Guo Hong An juge que les autres principes développés plus tard dans le temps, tels que "fidélité, fluidité, beauté», "ne rien ajouter ni réduire», "ressemblance à l'esprit», qui ont pu voir le jour à la lumière des principes de Yan Fu, sont, pour lui, moins saisissables, moins faciles à appliquer en toutes circonstances. Les principes de Yan Fu garderont leur vitalité à condition que l'on puisse les développer et y assigner de nouvelles significations (voir Guo Hong An et Xu Jun 1999: 204-207).

$\mathrm{Xu}$ Yuanchong, traducteur d'anglais et de français, considérant l'élégance comme la source même de la théorie de la traduction littéraire, propose sa théorie en trois «beautés» (d'esprit, de tonalité et de forme). En anglais, c'est «best words in best order». Best words se traduit par l'expression du contenu et des sons, tandis que best order par l'ordre de la beauté de forme et de sons (Xu Yuanchong et Xu Jun 1998: 202).

Essayons d'en tirer une conclusion: selon les traducteurs, l'activité de traduction ne doit pas être une activité aveugle, mais une activité qui s'accomplit en respectant des principes ou des critères. En Chine, les trois principes de Yan Fu sont acceptés, développés et ont été progressivement enrichis depuis un siècle malgré les divergences d'opinions. 


\subsection{Le processus de la traduction est aussi un processus de recherches}

Les recherches traductologiques dépassent largement, aujourd'hui, le niveau linguistique ou la comparaison linguistique. Quel est le processus de la traduction? Qu'est-ce qu'il faut faire avant la traduction? Si l'on traduit une œuvre, quelle est la procédure? Toutes ces questions sont exclusivement liées au processus même de la traduction. Vu l'importance de ce processus, nous avons eu le plaisir d'en discuter avec les traducteurs chinois lors de nos entretiens.

Depuis plus de 30 ans, de nombreux chercheurs portent particulièrement leur attention sur le processus de la traduction. Leur analyse se fait sous des angles différents. La théorie interprétative de l'École supérieure d'interprètes et de traducteurs de Paris (ESIT) constitue une première analyse. Se basant sur l'observation et l'analyse de l'interprétation professionnelle, les chercheurs de l'ESIT ont créé la théorie du sens, représentée par la trilogie du processus de l'interprétation: compréhension, déverbalisation et réexpression. L'attention des chercheurs a été, dès l'origine, centrée sur le processus de la traduction qui reflète bien naturellement les mécanismes intellectuels du traducteur, faisant appel aux résultats d'études neurologiques, neurolinguistiques, psycholinguistiques ainsi que d'autres disciplines concernant le cerveau humain et son développement. L'accumulation d'expériences réussies de la pratique et des analyses scientifiques les ont conduit à la théorisation de l'activité.

Le processus de la traduction peut se diviser, en théorie, en deux étapes principales: compréhension et expression. En revanche, il n'est pas aussi distinct qu'on ne le prétend dans la pratique. Ces deux étapes sont souvent croisées et influencées réciproquement. Cao Ying le justifie par son expérience. Il y a trois étapes dans sa traduction: A. prendre connaissance de l'œuvre, autrement dit, il la lit et relit à maintes reprises jusqu'à ce que les images du personnage apparaissent devant ses yeux; B. la traduire le plus fidèlement possible en chinois; C. d'abord la relire attentivement afin d'éviter tout ajout ou oubli, la modifier phrase par phrase pour voir si des erreurs s'y glissent; ensuite retoucher la traduction tout en respectant le génie de la langue chinoise dans le but de parvenir à une fluidité et à une facilité de compréhension. "Quelques fois je sollicite aussi de l'aide à mes amis acteurs dans la correction d'anomalies grâce à leur relecture...» (Cai Ying et Xu Jun 1999: 213).

Jiang Feng, traducteur de poèmes, montre sa désapprobation et parle d'une compréhension approfondie, ce qui veut dire que la compréhension demande non seulement au traducteur une connaissance linguistique du poème à traduire, mais une connaissance sur le poète, ses idées, son concept de valeur esthétique. C’est ainsi que Jiang Feng a essayé de pénétrer dans la vie, la pensée et l'époque où vivait le poète quand il traduisait Percy Bysshe Shelley... (Jiang Feng et Xu Jun 1998: 210).

Certains traducteurs parlent, pour leur part, de la nécessité de recherche sur l'œuvre originale avant que l'encre ne se dessine sur le papier. Lu Tongliu, traducteur d'œuvres littéraires italiennes nous décrit à sa façon le lien entre la traduction et les recherches: «la traduction littéraire ne peut jamais se passer des recherches littéraires... La traduction littéraire n'est pas une photocopie ou un fax de l'original. C'est un travail scientifique et créatif... Le traducteur doit être à la fois chercheur et écrivain» (Lu Tongliu et Xu Jun 1999: 207). Il ne manque pas l'occasion de nous montrer, à l'appui, une riche liste d'exemples précis relevés de ses publications. Li Mang, traducteur d'auteurs japonais, ne le contredit pas et reprend également ses exemples 
tout en les complétant par ses réflexions personnelles. La relecture minutieuse et le recul que le traducteur doit prendre après la traduction sont indispensables pour espérer une bonne traduction (Li Mang et Xu Jun 2000: 205).

La compréhension étant la première étape du processus, elle garde de plein droit sa place privilégiée dans la réalisation de la traduction. L'acte de lire et de comprendre, loin d'être objectif et passif, demande l'esprit créatif et la subjectivité du traducteur. Ye Junjian est convaincu que la compréhension, reposant sur la qualité du traducteur, sur son niveau culturel, esthétique et artistique, ne peut s'accomplir en une seule lecture (Ye Junjiang et Xu Jun 1998: 203).

La compréhension n'étant souvent pas la même d'un traducteur à l'autre, la retraduction d'une même œuvre en chinois, à la même époque que l'auteur ou à une autre époque, peut trouver sa raison d'être: les niveaux linguistiques et cognitifs du traducteur limitent sa compréhension, celle-ci a sûrement une conséquence sur la réexpression, d'où l'envie de retraduire de belles œuvres, d'où le processus sans fin de recherches.

\subsection{La traduction n'est pas une photocopie, elle est d'un caractère recréatif}

La traduction n'est qu'un transcodage de langue selon les profanes du métier. Contrairement à ce qu'ils croient, la démarche cognitive des traducteurs littéraires dépasse largement une telle simplicité linguistique. Quel lien peut-il y avoir entre l'original et la traduction? Celle-ci est plutôt une photocopie ou une recréation de l'original ? Les réponses des traducteurs chinois expérimentés nous amènent à une réflexion sur la valeur théorique.

En analysant le lien entre l'original et la traduction, nous nous interrogeons pour ainsi dire sur celui entre l'auteur et le traducteur, entre la traduction et la création. Une distinction entre la traduction littéraire et la création littéraire semble, à Fang Ping, tout à fait naturelle, car les deux disciplines appartiennent à des concepts artistiques différents. On ne nie pas que la traduction est basée sur l'original, que le traducteur se trouve de ce fait dans une position subordonnée à l'auteur, avec les mains liées; la création littéraire bénéficie de toute liberté possible dans la description du monde compris et vu par l'auteur. Quant au traducteur, il lui est impératif de posséder une sensibilité littéraire, une bonne compétence d'appréhension et de compréhension, une habileté et une compétence de marier sa particularité et son goût à ceux de l'auteur. Il lui est également important de transformer cette sensibilité artistique en motivation artistique afin de mettre ses idées sur le papier, en s'appuyant sur une compétence d'expression linguistique (Fang Ping et Xu Jun 1998: 203). Comme l'œuvre parle à la place de l'auteur, nous ne pouvons connaître les sentiments de l'auteur véhiculés par ses expressions qui dissimulent souvent le sens et l'esprit que l'auteur veut exprimer. Ceci dit, l'original linguistique n'est qu'un support à la traduction. Il ne faut cependant pas non plus perdre de vue que le processus de la traduction est un processus d'interprétation, au sens propre du terme: «il n'est pas du tout étonnant de voir qu'avec le temps, l'original se couvre de couleurs et de visages différents suite aux traductions, cela s'explique justement par le fait que la traduction n'est pas une photocopie, mais une recréation» (Ye Junjiang et Xu Jun 1998: 203). 
$\mathrm{Xu}$ Yuanchong prend particulièrement en considération la nature de recréation lorsqu'il s'agit de la traduction littéraire. "Le $\mathrm{XxI}^{\mathrm{e}}$ siècle sera une époque de littérature mondiale, la traduction littéraire doit devenir la littérature de traduction, en ce sens que des œuvres traduites doivent devenir elles-aussi les œuvres littéraires, de telle sorte qu'elles puissent faire partie de la littérature mondiale» (Xu Yuanchong et Xu Jun 1998: 202).

Selon Guo Moruo, une bonne traduction équivaut à une création littéraire, et même la dépasse quelquefois. La traduction littéraire ne se différencie pas de la création littéraire. La création et la traduction sont, pour lui comme la peinture. «La création prend la réalité comme modèle, la traduction prend de son côté l'œuvre originale, plus la réalité décrite dans l'original. Voilà le point commun entre la création et la traduction littéraires» (Xu Yuanchong et Xu Jun 1998: 202).

Guo Hong An dissèque le lien entre l'auteur et le traducteur du point de vue psychologique. D’après lui, il existe pour le traducteur trois positions vis-à-vis de l'auteur: supérieure, égale ou inférieure. La position supérieure pousse souvent le traducteur à transformer l'original, ajoutant ou supprimant des choses qui lui paraissent trop implicites en choisissant des métaphores propres à sa communauté linguistique, cela se fait souvent sous prétexte de l'insatisfaction du texte original ; la position égale aide à produire, chez le traducteur, un sentiment amical qui suscite ensuite chez lui une sensibilité et une compréhension facile, au point qu'il s'intègre assez rapidement à l'original et le traduit de façon harmonieuse ; la position inférieure incite le traducteur à ressentir un respect et une modestie. Il met par conséquent suffisamment de temps pour arriver à la compréhension du sens, du style, et de la façon par laquelle l'auteur s'exprime. En conjuguant tous ses efforts pour le parfait éventuel de la traduction, le traducteur humble prend toujours une grande précaution, en respectant l'auteur et en laissant la liberté à son lecteur plus intelligent que lui à ses yeux (voir Guo Hong An et Xu Jun 1999: 204-207).

Yang Wuneng avoue qu'en prenant position pour la théorie herméneutique, l'activité de traduction littéraire est par nature une interprétation, mais une interprétation particulière, littéraire et artistique, différente de l'interprétation au sens général du terme et comportant ses caractéristiques propres: A. interprétation globale et complète du texte; B. interprétation approfondie du contenu; C. interprétation visible et déductive en forme; D. interprétation active et dynamique chez le traducteur; E. interprétation relative et évolutive au sujet de l'original et de l'auteur. En résumé, l'interprétation générale est plus libre, tandis que celle de la traduction est limitée par le sens, la forme, la sonorité, les sentiments, le goût, la quantité, la qualité, etc., de l'original. Ceci dit, dans l'interprétation, il sera difficile de ne pas laisser au traducteur une certaine liberté de recréation, car le traducteur ne peut jamais perdre sa particularité et sa subjectivité, d'où la retraduction d'une même ouvre et son évolution avec le temps (Yan Wu Neng et Xu Jun 2000: 215).

Les cinq particularités de l'interprétation en traduction que décrit antérieurement Yang Wuneng nous aident beaucoup dans la compréhension de la nature de la traduction d'une part, dans la distinction entre la traduction générale et la traduction littéraire d'autre part. On pourrait proférer que c'est un approfondissement des réflexions sur la traduction littéraire et un principe important pour mesurer la liberté dans la traduction. 


\subsection{La subjectivité du traducteur}

"Un valet servant deux maîtres », ainsi décrit-on souvent la position embarrassante du traducteur, valet à la fois de l'original et du lecteur de la traduction. En réalité, le traducteur, si nous analysons objectivement son rôle, détient une liberté en lecture, en compréhension, en interprétation et en expression. Les traducteurs avec qui nos discussions ont lieu reconnaissent le rôle déterminant du traducteur pour le résultat de la traduction. Paradoxalement, la plupart des publications des recherches traductologiques en Chine ne traite que la technique de la traduction, sans nécessairement ni efficacement étudier la subjectivité du traducteur dont personne ne peut prétendre l'inexistence. Reconnaître au lieu de nier son rôle déterminant postule une étude plus approfondie sur les influences qu'exercent des facteurs autres que la linguistique, tels que les facteurs subjectifs et psychologiques, la personnalité, le niveau de connaissances générales, la compétence linguistique et la position du traducteur, les critères de valeurs etc., sur les produits de la traduction.

«La traduction littéraire est un processus chimique, en d'autres termes, un art de transformer l'original en texte traduit, un art de faire de la beauté originale la beauté reproduite et une méthode d'application» (Xu Yuanchong et Xu Jun 1998: 206). Xu Yuanchong a expliqué son point de vue en prenant un exemple à l'appui : la première phrase de «Qu Yuan», un des poèmes les plus connus écrits par Mao Tsédong, 《屈子当年赋楚骚》 peut être traduit littéralement par 《Qu Yuan», 《that year», «rhytm», «Sorrow after Departure». Mais «that year» n'est pas clair, trop de syllabes dans «Sorrow after Departure»... La traduction finale est "Qu Yuan had rhytmed his grief long long ago». Voilà une traduction «chimique». Luo Xinzhang y ajoute aussi en exemple la première phrase tirée de la traduction de Jean Christophe: «Le grondement du fleuve monte derrière la maison ».

La traduction littérale présentée ci-dessous utilise beaucoup plus de mots et est redondante:

“大 江的轰隆隆声, 从屋子后面 升上来”

grand fleuve de grondement bruit, de maison derrière lever monter?

La traduction de Fu Lei est recherchée, simple, mais d'un goût artistique en chinois :

“江 声浩荡, 自屋后上升”(Xu Jun, Luo Xinzhang, Shi Kanqiang et Yuan

Xiaoyi 199: 206)

(fleuve bruit vaste, de maison derrière monter)

Les exemples sont multiples, tous témoignent unanimement de l'existence de la subjectivité du traducteur. La vitalité d'une œuvre traduite dépend dans une certaine mesure de l'esprit créatif, de la personnalité et de la particularité du traducteur. Sans eux, «d'où viendrait la littérature, l'art et la création?» s'interroge Yang Wuneng. Sinon, le traducteur pourrait être un jour complètement remplacé par la traduction automatique de l'ordinateur. La subjectivité et l'esprit créatif du traducteur devraient pourtant aller de pair avec le respect pour l'auteur (Yang Wuneng et Xu Jun 2000: 214).

Les arguments développés par les traducteurs nous amènent à confirmer que l'existence de la subjectivité du traducteur est une réalité, elle aide en tout cas dans la compréhension et l'expression du traducteur. 


\subsection{La réexpression du style}

On ne pourrait pas parler de traduction littéraire sans parler de compréhension et d'expression du style original, puisque pour les traducteurs à qui nous nous adressions, le style est la vie artistique d'une œuvre littéraire, le négliger ou le trahir, c'est écorcher l'œuvre. Les traducteurs en sont théoriquement conscients et ont suffisamment de références que nous trouvons intéressantes et précieuses.

La définition du style en chinois recouvre des notions assez larges allant du général au concret: style de l'époque, style de la nation, style d'un chapitre, d'une expression ou d'un caractère... En un mot, le style renferme tous les facteurs liés au contenu et à la forme de l'œuvre, à l'auteur, à sa personnalité et aux caractéristiques de sa création. En traduction littéraire, c'est le style linguistique qui demande, nous l'avons constaté lors des entretiens, le plus d'énergie des traducteurs.

Lin Yi An trouve que le style de l'auteur est le plus difficile à rendre, qu'il s'agisse de langues de faible diffusion, telles que le portugais, ou de langues de forte diffusion, telles que l'anglais ou le français. Il n'est vraiment pas facile de saisir et de retransmettre de façon fidèle le style propre à l'auteur, quelquefois insaisissable. Ce qui importe, c'est de l'analyser et de retrouver ce que l'auteur veut exprimer à travers les mots, car le style linguistique reflète et incarne celui de l'auteur (Lin Yi An et Xu Jun 2000: 214).

Certains traducteurs ont une vision plus ample du style. Vu les différences linguistiques, un style sous une certaine forme peut être rendu sous une autre forme. Les gens de pays différents vivent en fin de compte sur la même planète, éprouvent les mêmes sentiments, ont les mêmes besoins, sauf qu'ils les expriment sous des formes différentes et de manière différente, c'est ce que postule Guo Hong An. Si ces différences ne peuvent se traduire en une autre langue, il est toujours possible d'en rendre le plus possible, au moins partiellement. L'intraduisibilité prétendue de certains poèmes et surtout de leur style n'empêche pas certains traducteurs performants et particulièrement habiles en langue d'arrivée d'achever parfaitement leur traduction grâce à leur passion pour la littérature. "Yang (masculin) et fermeté», "Yin (féminin) et souplesse» sont deux grandes catégories qu'on peut associer au style littéraire et à partir desquelles on peut tracer une longue liste de sous-catégories. Cette clarification donne accès à une meilleure compréhension et réexpression du style, à condition que le traducteur «se creuse» la tête dans ce travail non sans pénibilité (Guo Hongan et Xu Jun 1999: 205).

«Tout bon auteur a son style, qui évolue et s'améliore durant sa vie de création. En le lisant, nous ressentons fortement sa particularité qui se mélange rarement avec celle d'un autre... Quant à la retransmission de ce style, cela devient un problème assez complexe», indique Cao Ying, "J’insiste sur la compréhension et l'appréhension qui sont pour moi une condition préalable de reproduction. Sans compréhension, la reproduction reste dans le vide», poursuit-il (Cao Ying et Xu Jun 1999: 211-212).

Tu An le précise davantage en s’appuyant sur son expérience: «Je cherche, dans ma traduction poétique, aussi bien la beauté stylistique, esthétique que celle de la forme et de la rime... Il faut que le traducteur appréhende le sentiment qu'éprouve l'auteur lors de sa création à tel point que les deux esprits s'accordent et les deux âmes s'embrassent» (Tu An et Xu Jun 1999: 204). 
Le traducteur se sent souvent dépourvu et exposé aux obstacles face à la reproduction du style original. Les œuvres de William Faulkner caractérisées par «le courant de conscience» sont pour les Chinois, obscures, abstraites, redondantes, compliquées, et finalement difficiles à comprendre. Li Wenjun prend une attitude très active face à ces obstacles: la richesse du style de Faulkner présente beaucoup de difficultés pour le traducteur, mais elle est aussi un défi et lui offre une occasion de display (Li Wenjun et Xu Jun 2000 : 201). D'après lui, tout est traduisible en chinois, la pensée comme l'esprit. La pauvreté n'appartient pas à la langue chinoise, mais à la maîtrise insuffisante de la part du traducteur. La sensibilité, la compétence de pénétration, de distinction et de jugement face à une langue et un art sont les plus importants pour un traducteur (Li Wenjun et Xu Jun 2000: 204-205).

Basés sur leurs riches expériences, ces traducteurs chevronnés nous lèguent un patrimoine méthodologique par lequel on peut espérer une reproduction plus ou moins idéale du style littéraire, telle que lire des œuvres chinoises du même genre, du même sujet avant de prendre la plume; faire attention au lien entre l'ensemble de l'œuvre et ses parties...

Une constatation retient notre attention : le style du traducteur existe quelque part. Dans les œuvres traduites, on peut retrouver en fait le style de l'auteur mélangé à celui du traducteur. Si une œuvre est traduite en 10 versions, elle peut avoir 10 styles plus ou moins différents.

Il est alors possible d'en tirer trois conclusions: A. le style original doit être retransmis, mais imprégné inévitablement du style du traducteur; B. le style du traducteur ne peut exister que s'il n'affecte pas le style original; C. le style de l'auteur et celui du traducteur doivent s'accorder, de sorte que la particularité stylistique du traducteur devienne un facteur tout à fait positif dans la retransmission du style de l'auteur, ceci pour l'harmonie et l'union du style du texte d'origine et du texte traduit.

\subsection{La pensée imagée et la recréation des images}

La création littéraire est un art linguistique, la traduction l'est aussi. La pensée imagée est indispensable à leur réalisation. L'auteur se donne beaucoup de mal pour la création des personnages, il met en œuvre sa faculté d'imagination. Les lecteurs de générations différentes sont impressionnés par les images sculptées et façonnées dans les œuvres littéraires. Il en est de même quand il s'agit de la recréation de ces images dans la traduction. Autrement dit, le traducteur doit s'obliger et s'efforcer de reproduire les images construites, saisies à la lecture. "L'image du personnage est l'âme de l'œuvre littéraire.» Le talent de l'auteur se traduit même par la compétence de recréation d'images. «Tolstoï est maître en la matière. Pour le traduire, je lis et pénètre dans son ouvre pour que les images des personnages se reproduisent dans ma tête, ensuite, j'essaye de mobiliser et d'employer les procédés nécessaires pour la recréation des mêmes images dans le texte d'arrivée, afin que mon lecteur chinois en partage la même vision» (Cao Ying et Xu Jun 1999: 213).

L'un des critères de jugement de la traduction est de voir si les images recréées suscitent le même intérêt et la même réaction chez le lecteur de la langue cible. Cette recréation exige du traducteur, en premier lieu, la connaissance des langues de départ et d'arrivée; en second lieu, le génie et l'intelligence littéraires et la pensée imagée. Les expressions, les rimes, le rythme et la structure sont les premiers facteurs de 
jugement artistiques et esthétiques. Si le traducteur veut faire aimer le personnage recréé, il doit d'abord l'adorer lui-même, et le décrire ensuite dans une langue propre à lui-même. Lu Tongliu s'exclame après la traduction de Carméla: "J'ai fini par aimer Carméla, cette belle fille mais bien malheureuse»(Lu Tongliu et Xu Jun 1999: 208).

Il en est de même pour la traduction poétique. Les poèmes sont riches en images souvent décrites par métaphores. Jiang Feng n'est pas d'accord avec le changement d'image pour faciliter la réception du lecteur de la langue d'arrivée. Tu An a, de son côté, une opinion personnelle sur l'image: Si Hamlet sous la plume de Shakespeare est représenté par 1000 acteurs, il y aura autant de Hamlet. La transmission complète est impossible. Par contre, ces mille Hamlet doivent être le même prince danois de Shakespeare, «ne jamais démordre de ses principes malgré les mille changements apparents ». Cette règle est applicable à la traduction... (Tu An et Xu Jun 1999: 205).

Revenons de nouveau au principe de fidélité à l'original, nous dirons que le traducteur doit marier sa traduction aux recherches littéraires, essayer de maîtriser les caractères, particularités et qualités de l'auteur, ceci pour mieux connaître son personnage et mieux le redécrire.

\subsection{Le contenu et la forme, ressemblance à l'esprit et ressemblance à la forme}

Une œuvre n'est littéraire que lorsque sa forme impressionne plus le lecteur que son contenu, affirment certains. La forme joue un rôle considérable dans les produits littéraires: un sujet peut s'exprimer de plusieurs façons. Dans la littérature contemporaine, une œuvre littéraire peut faire sensation non par son contenu, mais par sa forme riche et nouvelle, citons par exemple Alain Robbe-Grillet et Marguerite Duras. La traduction de leurs œuvres est devenue en Chine un moteur dans l'évolution de la littérature du pays. En ce qui concerne la relation entre le contenu et la forme, Guo Hong An, traducteur de plusieurs ouvres de Camus, a insisté sur leur interdépendance en prenant comme exemple le style de Camus:

Albert Camus cherche un style "noble», style renfermant la dignité et la fierté de l'homme. Le style le plus noble est, à ses yeux, de montrer au mieux la révolte. Ce style noble ne reste pas au niveau purement et simplement formel, mais il permet aussi l'expression précise du contenu. Camus ne cherche point une forme sans se soucier du contenu, ce qu'il poursuit dans ses œuvres, c'est un lien constant et permanent entre le contenu et la forme... (Guo Hong An et Xu Jun 1999: 205).

La combinaison la plus parfaite entre contenu et forme constitue un défi pour le traducteur, en particulier dans la traduction poétique. Souvent, on rejette le contenu pour garder la forme ou au contraire, on sacrifie la forme pour le contenu. La mesure entre le contenu et la forme fait naître l'hésitation ou le doute qui, par la suite, prêtent le flanc à ceux qui sont pour l'intraduisibilité poétique. Pour Jiang Feng, la forme désigne la langue, non la prosodie:

Il est naturel, dans la traduction poétique, de rendre le style et le rythme. Or, il n'est pas question de traduire la prosodie. La même prosodie peut faire rédiger des poèmes complètement différents... La forme n'est pas purement et simplement le revêtement d'un poème, le conducteur d'information. Elle est, dans la plupart des cas, le contenu et le conducteur du message. La littérature poétique d'aujourd'hui en témoigne... (Jiang Feng et Xu Jun 1998: 207-210). 
Jiang Feng n'est pas solitaire dans son camp, un profond retentissement se produit parmi beaucoup d'autres traducteurs poétiques.

Li Mang s'aligne avec lui contre ceux qui, prenant le poème comme une peinture, croient qu'on peut mettre la forme dans «l'oubli» si «l'obtention» du contenu est possible. Il le rejoint pour dire ensuite que l'esprit est esclave de la forme, si celleci n'est pas précise, la vérité de l'esprit se perd. En ses termes, «il faut décrire l'esprit à l'aide de forme». Elle est décidée par le contenu, en revanche, l'expression du contenu réclame une forme qui s'y adapte (Li Mang et Xu Jun 2000 : 206-207).

Le débat sur le lien entre contenu et forme nous plonge dans des discussions sur la ressemblance à l'esprit et la ressemblance à la forme. Subjectivement parlant, le traducteur aimeraient conserver aussi bien la forme que l'esprit. Paradoxalement, il doit souvent trancher face à cette difficulté de choix. La traduction est plutôt une peinture qu'une photo: une même réalité peut être décrite par des phrases longues en une langue étrangère, mais par des phrases courtes en chinois, pourvu que le sens reste complet. Le peintre a le plein droit de rendre les bras plus longs avec une taille plus petite d'un personnage, puisque c'est sa façon de voir les choses. Il pourrait aller jusqu'à un traitement exceptionnel pour mieux tailler son personnage (Xu Jun, Luo Xinzhang, Shi Kangqiang et Yuan Xiaoyi 1998: 204).

Xiao Qian fait une distinction entre la traduction littéraire et la traduction des œuvres des hommes politiques. La traduction des œuvres de Mao Tsédong, par exemple, lui dicte une fidélité au contenu sans le moindre dérivé. La souplesse, appliquée dans la traduction littéraire, ne retrouve presque jamais sa place dans la traduction de documents, sous quelque forme que ce soit (Xiao Qian, Wen Jieruo et Xu Jun 1999: 210-211).

En résumé, il est idéal que le traducteur conserve à la fois le contenu et la forme (le style) de l'original, mais dans la pratique, les traducteurs se trouvent toujours dans une situation embarrassante et ils sont obligés de faire un choix entre la ressemblance à l'esprit et la ressemblance à la forme.

\subsection{La critique de la traduction}

La traduction littéraire a besoin de critique, de même qu'une pièce de théâtre attend la réaction du public pour une amélioration. Sans critique, on a du mal à comprendre le pourquoi du bon ou du mauvais. Elle est pratiquement un remède efficace soignant la traduction en vue de son développement. Aujourd'hui, la retraduction fait rage en Chine; Le rouge et le noir a une vingtaine de versions dont la moitié est d'une qualité médiocre ou mauvaise, ce qui porte atteinte non seulement à l'intérêt $\mathrm{du}$ lecteur, mais également et surtout au développement sain de la traduction. Nous étions très touchés, lors de nos échanges avec les traducteurs chinois, par leur amour et leur sollicitude pour la cause de la traduction et leur sens des responsabilités dans l'exercice de leur métier.

Tout en soutenant la retraduction intelligente et justifiée pour une meilleure qualité de la traduction, puisque les niveaux des traducteurs varient de l'un à l'autre, Ji Xianlin critique fermement le manque de sens des responsabilités de certains dans leur retraduction. L'attitude du traducteur est, lui paraît-il, primordiale dans le résultat de la traduction. La traduction est certes difficile, mais surmonter les difficultés constitue la tâche sacrée du traducteur (Ji Xianlin et Xu Jun 1998: 211). 
Li Mang, appréciant, d'une part, les résultats obtenus ces dernières années, montre du doigt d'autre part les tendances néfastes existant dans le milieu de la traduction en Chine. Des copies de traduction, des œuvres bâclées, le manque de critiques constructives : tout cela favorise certainement la diffusion des traductions médiocres. En parlant des œuvres traduites de bonne qualité, il souligne qu'elles ont aussi besoin de critiques vu que les erreurs ou l'imperfection sont inévitables. Il est regrettable de voir que rares sont ceux qui ayant un sens des responsabilités entreprennent une critique constructive pour ouvrir de nouvelles possibilités de traduction (Li Mang et Xu Jun 2000: 209).

La critique, ayant sa valeur directrice pour la pratique, est une des composantes indispensables de la traductologie. Comment procéder à la critique? Quels moyens et quelles méthodes peut-on utiliser? Comment mettre en valeur le rôle de la critique dans la traduction? À ces questions, nos interlocuteurs offrent des propositions, suggestions et conseils intéressants.

Fang Ping n'est pas pour la critique destructive qui attaque toujours la faute ou les erreurs dans la traduction et insiste sur la fonction constructive de la critique qui, d'après lui, a pour tâche principale d'assainir l'activité traduisante et d'ouvrir un nouvel espace de traduction (Fang Ping et Xu jun 1998: 205). La critique ne vise pas le simple fait du bon ou du mauvais de la traduction, elle a pour tâche principale d'offrir un univers plus ample et plus vaste à la traduction, d'où l'existence légale des traductions d'une œuvre littéraire. Il sera intéressant d'avoir plusieurs traductions d'une œuvre de valeur si elles ont été réalisées par des traducteurs sérieux. Cela fournit une possibilité de compléter les lacunes de l'un et d'éviter les erreurs ou pertes de l'autre (Yang Wuneng et Xu Jun 2000: 217).

Xiao Qian et Wen Jieruo, un couple de traducteurs, se rallient pour affirmer que la critique de la traduction sert l'accumulation culturelle favorisant l'amélioration de la qualité de la traduction. La critique leur permet de réaliser une deuxième version d'Ulysse qui, à leurs yeux, pourrait être remplacée un jour, et pourquoi pas, par une troisième version de la même œuvre. Ils forment leurs vœux pour la naissance d'une version toujours meilleure que la précédente (Xiao Qian,Wen Jieruo et Xu Jun 1999: 215).

L'objectivité est le fondement de la critique, on ne peut pas imposer un critère à certains ni obéir à d'autres sans raisons justifiées. Les débats sont nécessaires pour le fleurissement des opinions (voir Tu An et Xu Jun 1999: 208-209).

La critique, nous semble-t-il, doit garder son essence positive et rester constructive, aider le traducteur à former un bon projet de traduction, s'orienter vers les études de faisabilité, attirer l'attention de plus de gens sur la traduction et contribuer à son développement et enfin aux échanges culturels entre les nations différentes.

Outre les sujets ci-dessus mentionnés, les traducteurs ont encore soulevé des problèmes qui méritent notre attention. Chen Yuan, traducteur et linguiste, s'intéresse de son côté au lien entre la langue et la traduction, au problème de traduisibilité et de transformation linguistique; quant à Lin Yi An, il essaie de définir le concept d' «étranger» dans la traduction en se basant sur la traduction d'œuvres latinoaméricaines, tandis que Shi Kangqian pose le problème de la reproduction de l'exotisme. L'étendue de leur vision et la perspicacité de leurs pensées, difficiles d'être reproduites en une dizaine de pages, contribueront sans aucun doute au développement des recherches et à la pratique de la traduction en Chine. Leurs réflexions étant 
d'une grande valeur, elles constituent un patrimoine incomparable qu'ils lèguent à la cause de la traduction du pays. Nous sommes donc convaincus qu'elles pourront contribuer aux échanges littéraires, culturels entre la Chine et d'autres pays du monde.

\section{Conclusion}

Les discussions avec les grands traducteurs chinois nous ont non seulement permis d'étudier les problèmes qui se posent dans la pratique traduisante, mais aussi de constater que la théorisation de la traduction ne peut se passer des expériences réussies. Les 10 problèmes dont il est question dans la deuxième partie de cette présentation ne se bornent pas à traduire les soucis et préoccupations des traducteurs dans l'exercice de leur métier, mais offrent une valeur théorique non négligeable. L'énergie déployée dans ces travaux n'est qu'un premier pas de recherches théoriques sur la traduction littéraire, plus précisément, une nouvelle tentative pour la théorisation de la traduction littéraire, à partir de la pratique et des expériences.

\section{RÉFÉRENCES}

An, T. et X. Jun (1999) : "La fidélité, la clarté et l'élégance versus le vrai, le beau et le bon », Forêt de traduction 4, Nanjing, Éditions de Yilin, p. 203-209.

Feng, J. et X. Jun (1998) : «Harmonie entre la forme et l'esprit: un idéal de la traduction poétique », Forêt de traduction 6, Nanjing, Éditions de Yilin, p. 206-212.

Hongan, G. et X. Jun (1999): «Sur le style de la traduction », Forêt de traduction 3, Nanjing, Éditions de Yilin, p. 204-207.

JiN, B. (1996): «Pourquoi je traduis», Critique sur la traduction 1, Nanjing, Éditions de Yilin.

Jun, X., Xinzhang, L., Kangqiang, S. et Y. Xiaoyi (1998): «Sur la recréation dans la traduction littéaire », Forêt de traduction 1, Nanjing, Éditions de Yilin, p. 201-209.

Jun, X. (2001): Théories et pratique de la traduction littéraire: dialogues et réflexions, Nanjing, Éditions de Yilin.

Junjiang, Y. et X. Jun (1998): «L'œuvre traduite: pour sa qualitéde chef-d'œuvre», Forêt de traduction 5, Nanjing, Éditions de Yilin, p. 200-203.

MANG, L. et X. Jun (2000) : «La traduction: un art de recréer l'œuvre d'origine», Forêt de traduction 1, Nanjing, Éditions de Yilin, p. 203-209.

PING, G. et X. JUN (1998) : «Sur la compensation et la perte dans la traduction littéraire», Forêt de traduction 2, Nanjing, Éditions de Yilin, p. 202-206.

Qian, X., Jieruo, W. et X. Jun (1999) : «La traduction: une recréation artistique infinie», Forêt de traduction 1, Nanjing, Éditions de Yilin, p. 210-215.

Ruinong, Z., YI, Y. et X. Jun (1999): "Sur la traduction et la création", Forêt de traduction 2, Nanjing, Éditions de Yilin, p. 202-209.

Suru, S. (1998) : Fidélité, clarté et élégance: Études sur la théorie de Yan Fu, Beijing, Maison d'édition Shangwu.

Tongliu, L. et X. Jun (1999): «Pour une meilleure reproduction de la forme artistique de l'œuvre originale», Forêt de traduction 6, Nanjing, Éditions de Yilin, p. 206-211.

Wenjun, L. et X. Jun (2000) : «Sur les compétences du traducteur», Forêt de traduction 2, Nanjing, Éditions de Yilin, p. 200-206

Wuneng, Y. et X. Jun (2000): «Sur le sujet traduisant dans la traduction littéraire», Forêt de traduction 3, Nanjing, Éditions de Yilin, p. 213-220.

Xianlin, J. et X. Jun (1998): «Sur le grand rôle que joue la traduction», Forêt de traduction 4, Nanjing, Éditions de Yilin, p. 209-211.

Yi AN, L. et X. Jun (2000) : «Sur la traduction et la réception de la littérature américano-latine en Chine», Forêt de traduction 5, Nanjing, Éditions de Yilin, p. 212-219 
804 MetA, XLIX, 4, 2004

YING, C. et X. JUn (1999) : «Soyons un homme sincère et un traducteur sérieur », Forêt de traduction 5, Nanjing, Éditions de Yilin, p. 209-213.

Yuan, C. et X. Jun (2000): «Sur la traduction et la langue», Forêt de traduction 4, Nanjing, Éditions de Yilin, p. 207-213.

Yuanchong, X. et X. Jun (1998): «Sur la traduction: un art d'embellir», Forêt de traduction 3, Nanjing, Éditions de Yilin, p. 201-207. 